\title{
Retraction Note to: Optical properties of rare earth-doped barium aluminate synthesized by different methods-a review
}

\author{
Jagjeet Kaur ${ }^{1} \cdot$ Beena Jaykumar ${ }^{1} \cdot$ Vikas Dubey $^{1} \cdot$ Ravi Shrivastava ${ }^{1}$. \\ N. S. Suryanarayana ${ }^{1}$
}

Published online: 17 August 2019

(c) Springer Nature B.V. 2019

\section{Retraction Note to: Res Chem Intermed (2015) 41:2317-2343 https://doi.org/10.1007/s11164-013-1349-z}

The Editor-in-Chief has retracted this article [1] because it contains material that substantially overlaps with the following articles (amongst others) [2-5]. In addition, Figures 4-17 have been reproduced from other sources without permission. Jagjeet Kaur, Vikas Dubey and N. S. Suryanarayana agree with this retraction. Beena Jaykumar and Ravi Shrivastava have not responded to any correspondence about this retraction.

\section{References}

1. J. Kaur, B.J.V. Dubey, S. Ravi, N.S. Suryanarayana, Res. Chem. Intermed. 41(4), 2317 (2015)

2. L.C.V. Rodrigues, R. Stefani, H.F. Brito, M.C.F.C. Felinto, J. Hölsä, M. Lastusaari, T. Laamanen, M. Malkamäki, J. Solid State Chem. 183(10), 2365 (2010)

3. Y. Lin, Z. Zhang, J.Z. ZilongTang, Z. Zheng, L. Xiao, Mater. Chem. Phys. 70(2), 156 (2001)

4. R. Stefani, L.C.V. Rodrigues, C.A.A. Carvalho, M.C.F.C. Felinto, H.F. Brito, M. Lastusaari, J. Hölsa, Opt. Mater. 31(12), 1815 (2009)

5. H.F. Brito, Maria C.F.C. Felinto, J. Hölsä, T. Laamanen, M. Lastusaari, M. Malkamäki, P. Novák, Lucas C.V. Rodrigues, R. Stefani, Opt. Mater. Express 2(4), 420 (2012)

Publisher's Note Springer Nature remains neutral with regard to jurisdictional claims in published maps and institutional affiliations.

The original article can be found online at https://doi.org/10.1007/s11164-013-1349-z.

Beena Jaykumar

beenajaykumar@gmail.com

1 Department of Physics, Government Vishwanath Yadav Tamaskar Post Graduate Autonomous College, Durg, India 